Louisiana State University

LSU Digital Commons

Faculty Publications

Department of Physics \& Astronomy

$1-1-2002$

\title{
Space-Time Considerations in the Phase Locking of High Harmonics
}

Mette B. Gaarde

Lunds Tekniska Högskola

Kenneth J. Schafer

Lunds Tekniska Högskola

Follow this and additional works at: https://digitalcommons.Isu.edu/physics_astronomy_pubs

Recommended Citation

Gaarde, M., \& Schafer, K. (2002). Space-Time Considerations in the Phase Locking of High Harmonics. Physical Review Letters, 89 (21) https://doi.org/10.1103/PhysRevLett.89.213901

This Article is brought to you for free and open access by the Department of Physics \& Astronomy at LSU Digital Commons. It has been accepted for inclusion in Faculty Publications by an authorized administrator of LSU Digital Commons. For more information, please contact ir@lsu.edu. 


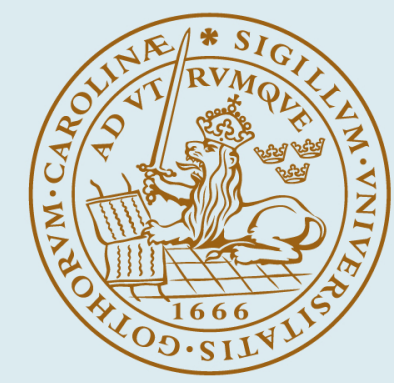

\title{
LUND UNIVERSITY
}

\section{Space-time considerations in the phase locking of high harmonics}

\author{
Gaarde, Mette; Schafer, Kenneth
}

Published in:

Physical Review Letters

DOI:

10.1103/PhysRevLett.89.213901

2002

Link to publication

Citation for published version (APA):

Gaarde, M., \& Schafer, K. (2002). Space-time considerations in the phase locking of high harmonics. Physical Review Letters, 89(21). https://doi.org/10.1103/PhysRevLett.89.213901

\section{Total number of authors:}

2

\footnotetext{
General rights

Unless other specific re-use rights are stated the following general rights apply:

Copyright and moral rights for the publications made accessible in the public portal are retained by the authors and/or other copyright owners and it is a condition of accessing publications that users recognise and abide by the legal requirements associated with these rights.

- Users may download and print one copy of any publication from the public portal for the purpose of private study or research.

- You may not further distribute the material or use it for any profit-making activity or commercial gain

- You may freely distribute the URL identifying the publication in the public portal

Read more about Creative commons licenses: https://creativecommons.org/licenses/

Take down policy

If you believe that this document breaches copyright please contact us providing details, and we will remove

access to the work immediately and investigate your claim.
} 


\title{
Space-Time Considerations in the Phase Locking of High Harmonics
}

\author{
Mette B. Gaarde ${ }^{1}$ and Kenneth J. Schafer ${ }^{1,2,3}$ \\ ${ }^{1}$ Department of Physics, Lund Institute of Technology, P.O. Box 118, S-22100 Lund, Sweden \\ ${ }^{2}$ Niels Bohr Institute, Ørsted Laboratory, Universitetsparken 5, 2100 Copenhagen, Denmark \\ ${ }^{3}$ Department of Physics and Astronomy, Louisiana State University, Baton Rouge, Louisiana 70803-4001
}

(Received 16 May 2002; published 31 October 2002)

\begin{abstract}
The combination of several high order harmonics can produce an attosecond pulse train, provided that the harmonics are locked in phase to each other. We present calculations that evaluate the degree of phase locking that is achieved in argon and neon gases interacting with an intense, $50 \mathrm{fs}$ laser pulse, for a range of macroscopic conditions. We find that phase locking depends on both the temporal and the spatial phase behavior of the harmonics, as determined by the interplay between the intrinsic dipole phase and the phase matching in the nonlinear medium. We show that, as a consequence of this, it is not possible to compensate for a lack of phase locking by purely temporal phase manipulation.
\end{abstract}

DOI: 10.1103/PhysRevLett.89.213901

PACS numbers: 42.65.Ky, 32.80.Wr, 42.50.Hz

There are currently two distinct approaches to attosecond metrology based on high harmonic generation. The first, which aims to produce single $\simeq 500$ as $(1$ as $=$ $1 \times 10^{-18} \mathrm{~s}$ ) pulses, uses the cutoff region of the continuous harmonic spectrum generated by a few-cycle laser pulse [1]. The second route uses many-cycle driving pulses to generate a series of spectrally separated plateau harmonics which are then filtered and combined [2,3]. In this type of experiment, the degree of phase locking that can be achieved between the superposed harmonics determines whether or not an attosecond pulse train is produced [4]. Recently, Paul et al. [5] combined the five odd harmonics 11 through 19 generated in argon by a $40 \mathrm{fs}$ laser pulse to produce a train of 250 as pulses, and even shorter pulses could potentially be produced through the phase locking of more harmonics. The ability to produce shorter pulses depends crucially on the answer to two questions: (i) Are there macroscopic conditions that facilitate the phase locking of large numbers of harmonics, and (ii) can the individual harmonic phases be manipulated to force phase locking?

Most of the discussion of attosecond pulse trains has centered on the necessity for temporal phase locking the phase difference between neighboring harmonics must be constant at any given time during the pulse [26]. From the spectral point of view, this requires that all the harmonics in the superposition have the same frequency chirp. Since the harmonics are produced by the interaction of a focused laser pulse with a threedimensional nonlinear medium, the phase of each harmonic can also vary over its radial profile, leading to a spatial chirp [7]. In order to achieve phase locking, this spatial chirp must also be the same for all the contributing harmonics. In this Letter, we demonstrate that a lack of phase locking is always the result of both the temporal and the spatial phase behavior of the harmonics. As these are determined by the phase matching that results from the interplay between the microscopic and the macro- scopic phase contributions in a given configuration, rapid phase variations in one domain are accompanied by the same in the other domain. We show that, as a consequence of this, harmonics cannot be forced to phase lock simply via manipulation of their temporal phase behavior.

To study the effects of the harmonic spatiotemporal phase behavior on phase locking, we have carried out three-dimensional calculations including both the single atom and macroscopic response of rare gas atoms to an intense, focused $810 \mathrm{~nm}$ laser pulse by direct numerical integration of the time-dependent Schrödinger equation and subsequently solving the Maxwell wave equation in the slowly varying envelope approximation (SVEA) [810]. We explore conditions similar to those presented in [5] for argon, and their extension to the superposition of larger numbers of harmonics in neon. We calculate the radial profile of each harmonic at the exit of the medium, $E_{q}\left(r, t^{\prime}\right)=\mathcal{E}_{q}\left(r, t^{\prime}\right) e^{i \phi_{q}\left(r, t^{\prime}\right)}$. At a given time $t^{\prime}$ during the pulse, we construct the sub-laser-cycle time profile of the emission from the combination of odd harmonics $n$ through $m$ as

$$
I_{\mathrm{XUV}}\left(t, t^{\prime}\right)=\int r d r\left|\sum_{q=n}^{m} \mathcal{E}_{q}\left(r, t^{\prime}\right) e^{-i\left[q \omega_{1} t-\phi_{q}\left(r, t^{\prime}\right)\right]}\right|^{2} .
$$

In this equation $\omega_{1}$ is the laser frequency, and $t$ refers to the rapid variation of the electric field within one cycle of the laser field. In the results presented below, we have chosen $t^{\prime}$ to be at the peak of the driving laser field and will refer to the time profile as $I_{\mathrm{XUV}}(t)$ [11]. This time profile includes the effects of the spatial distribution of the harmonics and their phases through the integration over the radial coordinate.

In the limit where the contributing harmonics are exactly phase locked and of the same strength at all radii, $I_{\mathrm{XUV}}(t)$ is a periodic function of time with one narrow peak every half cycle of the laser field (see [4] for details). The Fourier transform-limited full width at half 
maximum (FWHM) duration of each subcycle pulse is determined by the number $N$ of odd harmonics included in $I_{\mathrm{XUV}}(t), \tau_{N} \approx T / 2 N$, where $T$ is the laser period. The complicated single atom phase behavior, in combination with phase matching and effects of ionization, often results in harmonics that are not phase locked, and the corresponding time profiles can exhibit both multiple peaks per half cycle and structures much longer than $\tau_{N}$. This is illustrated in Fig. 1 where we display the normalized time profile $I_{\mathrm{XUV}}(t)$ for two different phasematching configurations of the combination of ten harmonics $\left(\tau_{N}=135\right.$ as $)$ generated in neon by a $50 \mathrm{fs}$ driving laser pulse. We show two different relative positions of the laser focus and the center of the gas jet. When the laser is focused before the center of the jet, the time profile is a train of pulses, each of duration 170 as, with an excellent signal to noise ratio. In contrast to this, when the laser is focused into the center of the gas jet, the time profile has several peaks per half cycle which have merged into very broad structures.

To systematically study the influence of the macroscopic conditions on the attosecond time profile, we use $I_{\mathrm{XUV}}(t)$ to define a measure of how well phase locked a number of harmonics are. We calculate the ratio $\gamma_{t}$ between how much radiation is emitted within $\tau_{N}$ and all the radiation emitted during one half cycle:

$$
\gamma_{t}=\frac{\int_{\tau_{N}} d t I_{\mathrm{XUV}}(t)}{\int_{T / 2} d t I_{\mathrm{XUV}}(t)}
$$

We then normalize $\gamma_{t}$ to $\tilde{\gamma}_{t}=\left(\gamma_{t}-2 \tau_{N} / T\right) /$ $\left(0.775-2 \tau_{N} / T\right)$, where 0.775 is the ratio one would get from a Fourier transform-limited pulse. This means that if the harmonics are perfectly phase locked $\tilde{\gamma}_{t}$ equals

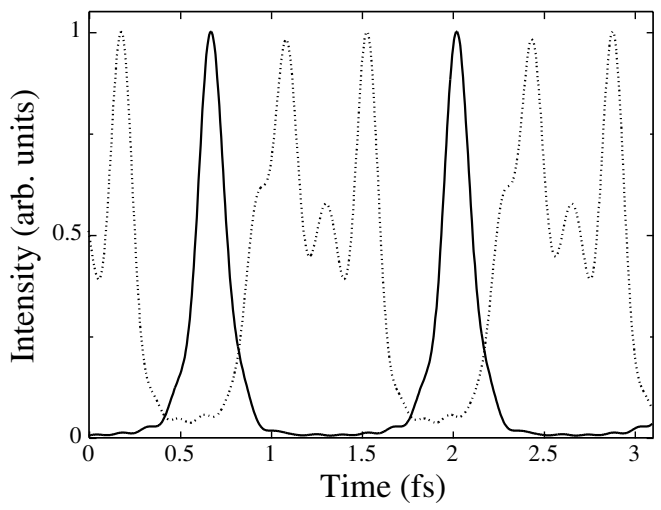

FIG. 1. Normalized time profiles of the combination of the odd harmonics 41 through 59 generated in neon by a 50 fs driving laser pulse with peak intensity $6 \times 10^{14} \mathrm{~W} / \mathrm{cm}^{2}$. The laser beam is loosely focused into a $1 \mathrm{~mm}$ gas jet in which the pressure is 5 Torr, with a confocal parameter $b=10 \mathrm{~mm}$. We show two different relative positions $z_{0}$ of the laser focus and the center of the gas jet, $z_{0}=-0.45 b$ (solid line) and $z_{0}=0$ (dotted line). one, and $\tilde{\gamma}_{t}$ is zero if $I_{\mathrm{XUV}}(t)$ is a constant. $\tilde{\gamma}_{t}$ has relevance for experiments since it measures the concentration of energy in a short time-interval $\tau_{N}$ during each half cycle, thus probing whether a train of attosecond pulses has been produced.

In Fig. 2, we plot $\tilde{\gamma}_{t}$ as a function of $z_{0}$. We first study conditions very similar to those described in [5]. We combine the odd harmonics 11 through $19\left(N=5, \tau_{N}=\right.$ 270 as) generated in argon by a 50 fs laser pulse with a peak intensity of $2.5 \times 10^{14} \mathrm{~W} / \mathrm{cm}^{2}$. The confocal parameter of the laser beam is $b=35 \mathrm{~mm}$, and the pressure in the $10 \mathrm{~mm}$ gas jet is 10 Torr. Evidently, there is good phase locking for a range of focus positions close to $-b / 2$. We find very good agreement with the experimental results; in particular, when $z_{0}$ is close to $-0.4 b$ the FWHM of each pulse in the time profile is 270 as (compared to an estimated 250 as in the experiment).

Also shown in Fig. 2 is a calculation of the phase locking of ten harmonics in neon using the same parameters as in Fig. 1, which could lead to the production of 135 as pulses. Again, good phase locking can be achieved over a range of $z_{0}$ close to $-b / 2$, i.e., when the laser is focused approximately one Rayleigh length before the center of the gas jet. We have found this to be true even when combining other harmonics (for example, 19-27 in argon, 41-49 in neon, 51-59 in neon) and/or using slightly different intensities and pressures. Our results thus seem to indicate that there are generic macroscopic conditions that, in general, lead to good phase locking. We find that it is important that all the superposed harmonics belong to the plateau region, where their phase behavior with respect to the laser intensity is similar. For example, in Fig. 2, the 59th harmonic in neon enters the cutoff region of the harmonic spectrum when $z_{0} \leq-0.43 b$ which leads to the rapid drop of $\tilde{\gamma}_{t}$ for $z_{0}<-b / 2$. It is also typical that phase locking is poor when $z_{0}$ is close to and larger than zero [12].

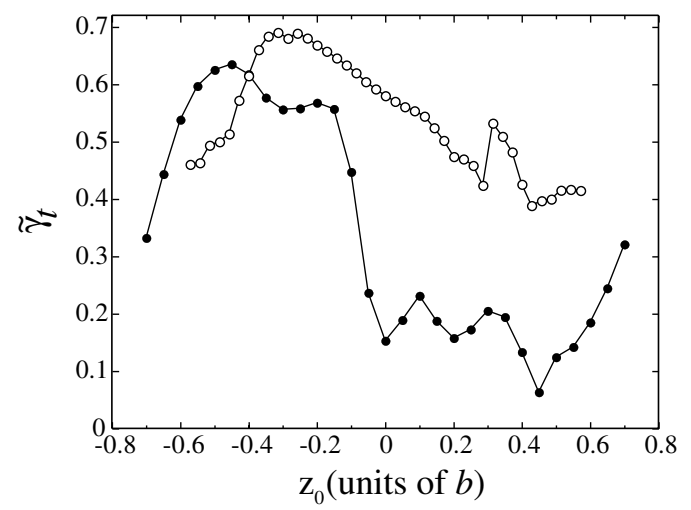

FIG. 2. Measure of phase locking as a function of the relative position $z_{0}$ of the laser focus and the center of the gas jet. $\tilde{\gamma}_{t}$ is shown for the odd harmonics 41-59 in neon (black circles), and 11-19 in argon (white circles). 
We now turn to the question of measuring and manipulating the harmonic phases. Since it is not yet possible to experimentally measure $I_{\mathrm{XUV}}(t)$, an indirect probe of phase locking was used in [5], where the (radially and temporally averaged) phase difference between adjacent harmonics was measured. In Fig. 3, we illustrate a similar measure for the time profiles shown in Fig. 1. We define the radially averaged phase difference $\overline{\Delta \phi}_{q+1}$ between neighboring harmonics $q$ and $q+2$ as

$$
\overline{\Delta \phi}_{q+1}=\frac{\int r d r\left[\phi_{q+2}(r)-\phi_{q}(r)\right] \mathcal{E}_{q+2}(r) \mathcal{E}_{q}(r)}{\int r d r \mathcal{E}_{q+2}(r) \mathcal{E}_{q}(r)} .
$$

That $\overline{\Delta \phi}_{q+1}$ provides a useful measure of the temporal coherence can be appreciated by comparing Figs. 1 and 3 . The phase differences in the $z_{0}=0$ case vary significantly from pair to pair, whereas they vary much less when $z_{0}=-0.45 b$. The approximately linear variation of the phase difference in the latter case corresponds to a quadratic variation of the spectral phase of the superposition of harmonics. Such a linear chirp is in general destructive for phase locking, and it is only because it is small in this case that the harmonics still phase lock.

It is worth stressing that a constant phase difference $\overline{\Delta \phi}_{q+1}$ does not guarantee phase locking. The spatial distortion of the harmonic wave fronts, which is not accounted for in a radially averaged phase measurement, can have a profound effect on the attosecond pulse train structure. A simple example of this is the superposition of harmonics with constant phases and only pairwise spatial overlap. This would still yield the same type of signal as measured in [5]; however, the lack of spatial overlap between all the harmonics gives rise to very broad peaks in the time profile.

The manipulation of phase locking is even more problematic than its measurement, because of the close connection between the spatial and temporal phase behaviors

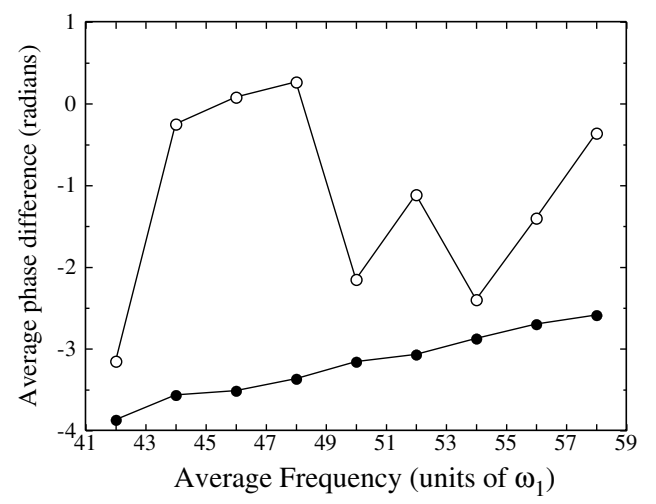

FIG. 3. Radially averaged phase difference, as defined in Eq. (3), between pairs of odd harmonics 41-59 in neon. The configurations $z_{0}=-0.45 b$ and $z_{0}=0$ are shown with black circles and white circles, respectively. of the harmonics. The possibility of imposing phase locking has been raised by Paul et al. [5], who speculate that their method for the measurement of harmonic phase differences could be used to subsequently properly phase all of the harmonics generated in a gas cell, yielding subpulses as short as 10 as. To study this suggestion, we return again to the two time profiles shown in Fig. 1. In Fig. 4, we plot their radial dependence, i.e., we plot the nearfield profile multiplied by the radial coordinate $r$, which is the integrand of Eq. (1). In the good phaselocking case $\left(z_{0} \approx-b / 2\right)$, the radial profile of the superposition is very regular and the harmonics phase lock simultaneously at all contributing radii. In the poor phase-locking case $\left(z_{0}=0\right)$, the radial profile of the superposition is very irregular with contributions from several different radii [13]. The harmonics do not phase lock at any radius, and the time profiles at different radii peak at different times. In order to induce phase locking, it would be necessary to compensate a different spectral phase at each radial position. Thus, a purely temporal manipulation of the harmonic phases cannot lead to phase locking in this case.

We have systematically tested the proposition that compensating the radially averaged phase difference between harmonics is insufficient to force phase locking by simulating such a temporal manipulation numerically. We have done a series of calculations in neon for various focusing conditions where we compensated for the radially averaged phases of the harmonics (as would be measured in an experiment that probes the timedependent harmonic frequencies [14]). Calculating $\tilde{\gamma}_{t}$ and comparing to the uncompensated cases, we find no improvement on the phase-locking measure shown in Fig. 2.

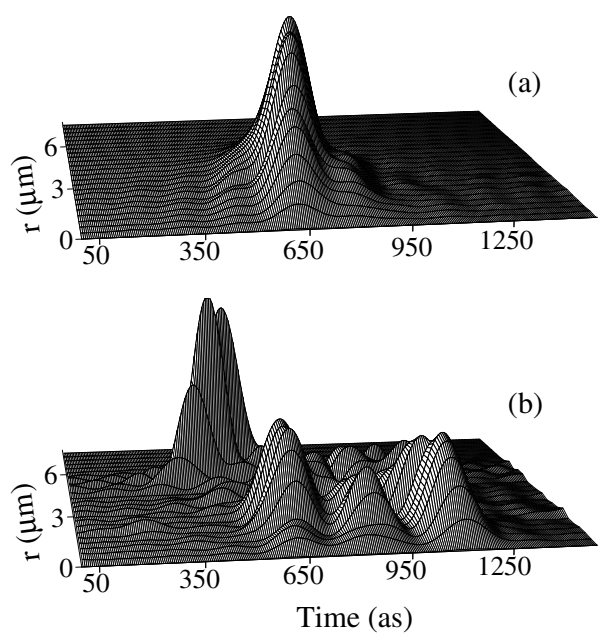

FIG. 4. Radial distribution of the time profile of the combination of the odd harmonics 41-59 in neon [integrand in Eq. (1)] for the two focusing positions (a) $z_{0}=-0.45 b$, and (b) $z_{0}=0$. 
The physics behind the failure of purely temporal manipulation of the harmonic phases can be understood via the intensity dependence of the harmonic generation process. When a superposition of several harmonics is not phase locked in time at some radius, it is because different harmonics exhibit different intensity-dependent behaviors as they propagate through the nonlinear medium. This leads directly to the fact that the harmonics have different radial phase behaviors through the radial variation of the driving laser intensity. Ultimately, this is because phase matching has not imposed a systematic phase behavior by, for example, preferring a specific single atom phase dependence over all others $[4,15]$. Thus, it is a general feature of harmonic generation that the imposition of phase locking is complicated by spatial effects which are inevitable when temporal phase locking is lacking to begin with.

By this same argument, when the radial profile of a superposition of several harmonics is well behaved, it is likely that they are phase locked. We therefore expect that phase-matching configurations which give nice radial profiles for the individual harmonics when focusing into the center of the gas jet (as measured in the experiment of Nisoli et al. [16]) are more likely to result in phase locking for $z_{0} \approx 0$ than the configuration discussed above [5]. Our results have been obtained using the SVEA $[8,10]$ and therefore do not necessarily generalize to few-cycle driving pulses $[1,17,18]$. Even beyond the SVEA, however, strongly distorted radial profiles signify different phase matching of the harmonic radiation at different radii, which is problematic for subfemtosecond pulse generation.

In summary, we have studied attosecond pulse train generation via the phase locking of several high order harmonics in argon and neon gases. We find a range of conditions in which the production of attosecond pulse trains is robust. In these conditions, both the temporal and the spatial phase coherence between the superposed harmonics is high, as expected. We have demonstrated that a full description of phase locking must take account of the spatial as well as the temporal phase variations of the harmonics. In particular, we have shown that to induce phase locking it is not sufficient to measure and subsequently compensate the spatially averaged temporal phase of each harmonic.

We thank A. LHuillier for valuable discussions, and P. Agostini for providing experimental details of Ref. [5]. This work was supported by the Swedish Natural Science Research Council. K. S. acknowledges the support of the Swedish Foundation for International Cooperation in Research and Higher Education (STINT), the Danish-American Fulbright Commission, and the National Science Foundation through Grant No. PHY-
9733890. Computer time was provided by the National Supercomputer Centre in Sweden.

[1] M. Hentschel et al., Nature (London) 414, 509 (2001); M. Drescher et al., Science 291, 1923 (2001).

[2] S. Harris, J. J. Macklin, and T.W. Hänsch, Opt. Commun. 100, 487 (1993).

[3] G. Farkas and C. Toth, Phys. Lett. A 168, 447 (1992).

[4] Ph. Antoine, A. L'Huillier, and M. Lewenstein, Phys. Rev. Lett. 77, 1234 (1996).

[5] P. M. Paul et al., Science 292, 1689 (2001).

[6] K. J. Schafer and K. C. Kulander, Phys. Rev. Lett. 78, 638 (1997).

[7] M. B. Gaarde, F. Salin, E. Constant, Ph. Balcou, K. J. Schafer, K. C. Kulander, and A. LHuillier, Phys. Rev. A 59, 1367 (1999).

[8] A. L'Huillier, P. Balcou, S. Candel, K. J. Schafer, and K. C. Kulander, Phys. Rev. A 46, 2778 (1992).

[9] K. C. Kulander, K. J. Schafer, and J. L. Krause, in Atoms in Intense Laser Fields, edited by M. Gavrila (Academic, San Diego, 1992).

[10] M. B. Gaarde and K. J. Schafer, Phys. Rev. A 65, 031406(R) (2002).

[11] In the SVEA, each $t^{\prime}$ during the pulse represents an independent propagation configuration. If the relevant harmonics are not temporally phase locked, they will have very different (spectral) phases at most times. Conversely, if the harmonics are phase locked in time, they are phase locked at all points in time. We have checked that the specific choice of $t^{\prime}$ is not important for our results.

[12] This finding is in contrast with the prediction of [4], which used dipole moments calculated in the strong field approximation and found that good phase locking could be achieved with $z_{0}=0$ as well as with $z_{0} \approx-b / 2$.

[13] This is not due to the fact that the intensity in the gas jet is lower for $z_{0}=-0.45 b$ than for $z_{0}=0$. The phase behavior when focusing symmetrically on the other side of the gas jet $\left(z_{0}=+0.45 b\right)$ is even more irregular than for $z_{0}=0$.

[14] E. Hertz et al., Phys. Rev. A 64, 051801(R) (2001); J. Norin et al., Phys. Rev. Lett. 88, 193901 (2002); T. Sekikawa, T. Katsura, S. Miura, and S. Watanabe, Phys. Rev. Lett. 88, 193902 (2002).

[15] D. G. Lee, H. J. Shin, Y. H. Cha, K. H. Hong, J.-H. Kim, and C. H. Nam, Phys. Rev. A 63, 021801(R) (2001); D. B. Milošević and W. Becker, Phys. Rev. A 62, 011403(R) (2000); D. G. Lappas and A. LHuillier, Phys. Rev. A 58, 4140 (1998).

[16] M. Nisoli et al., Phys. Rev. Lett. 88, 033902 (2002).

[17] I. P. Christov, R. Bartels, H. C. Kapteyn, and M. M. Murnane, Phys. Rev. Lett. 86, 5458 (2001).

[18] X. Chu and Shih-I Chu, Phys. Rev. A 64, 021403(R) (2001). 